\title{
Desenvolvimento de um método para determinação de ácidos graxos utilizando a espectroscopia no infravermelho com transformada de Fourier
}

\section{Development of a method for determination of fatty acid using FT- IR spectroscopy}

\author{
Dimas Augusto Morozin Zaia ${ }^{1}$; Leonardo de Oliveira Guarnieri ${ }^{2}$; Karina Nicole \\ Sobota $^{3}$; Henrique de Santana ${ }^{4}$, Cássia Thaïs Bussamra Vieira Zaia ${ }^{5}$
}

\section{Resumo}

No presente trabalho foi desenvolvida uma metodologia para a determinação de ácidos graxos em meios biológicos utilizando a espectroscopia no infravermelho com transformada de Fourier. Este método não precisa de reagente cromóforo ou pré-preparo da amostra. $\mathrm{O}$ ácido palmítico foi escolhido como padrão, pois é o ácido graxo encontrado em diversos meios biológicos. O espectro infravermelho do ácido palmítico mostrou duas bandas de absorção na região de 2852 e $2920 \mathrm{~cm}^{-1}$ devido ao estiramento $\mathrm{CH}$. Os resultados mostraram que estas bandas seguem a lei de Beer-Lambert numa ampla faixa de concentração de ácido palmítico (14 a $\left.257 \mathrm{mmol} \mathrm{L}^{-1}\right)$. Como padrão interno foi utilizado o ferricianeto de potássio $\left(\mathrm{K}_{3}\left[\mathrm{Fe}(\mathrm{CN})_{6}\right]\right)$. Diversos interferentes foram testados e somente o colesterol, o cloreto férrico (maior concentração), a mistura de aminoácidos para a banda em $2919 \mathrm{~cm}^{-1}$ (maior concentração) e o triacilglicerol mostraram-se interferentes ao método quando em concentração elevada. A nova metodologia, portanto, é vantajosa por ser de baixo custo e de fácil realização.

Palavras-chave: Ácidos graxos. Ácido palmítico. Meios biológicos. Espectroscopia no infravermelho.

\begin{abstract}
In the present paper a new methodology has been developed for determination of fatty acids in biological systems using FT-IR spectroscopy. For this method is not necessary chromophore reagent or pre sample preparation. Palmitic acid was chosen as standard, because it is found in several biological systems. The FT-IR spectrum of palmitic acid showed two absorption bands in the region of 2852 and 2920 $\mathrm{cm}^{-1}$ attributed to $\mathrm{CH}$ stretching. The results for these bands showed that the Beer-Lambert Law was followed in wide range of concentration of palmitic acid $\left(14\right.$ to $\left.257 \mathrm{mmol} \mathrm{L}^{-1}\right)$. Potassium ferricyanide $\left(\mathrm{K}_{3}\left[\mathrm{Fe}(\mathrm{CN})_{6}\right]\right)$ was used as internal standard. Several interferents were tested and only cholesterol, ferric chloride (higher concentration), mixture of amino acids for the band at $2919 \mathrm{~cm}^{-1}$ (higher concentration) and triglyceride could be interferent if they appear in high concentration. Thus, this new methodology has advantage to be not expensive and simple.

Keywords: Fatty acids. Palmitic acid. Biological systems. FT-IR Spectroscopy.
\end{abstract}

1 Professor Associado-C, Departamento de Química-CCE, Universidade Estadual de Londrina

2 Bacharelando em Biomedicina, Departamento de Ciências Fisiológicas-CCB, Universidade Estadual de Londrina

3 Bacharelando em Biomedicina, Departamento de Ciências Fisiológicas-CCB, Universidade Estadual de Londrina.

4 Professor Associado-C, Departamento de Química-CCE, Universidade Estadual de Londrina

5 Professora Associada-C, LaFiNeM-Laboratório de Fisiologia Neuroendócrina e metabolismo, Departamento de Ciências Fisiológicas-CCB, Universidade Estadual de Londrina. Corresponding author Cássia Thais B. V. Zaia e-mail: ctbvzaia@uel.br 


\section{Introdução}

Diversas metodologias têm sido desenvolvidas para a determinação de ácidos graxos, tais como: potenciometria (TURYAN; BEREZIN; SHENHAR, 1996); infravermelho próximo (ZHANG; LEE, 1997; MAN; MOH, 1998); fluorescência (STORCH; LECHENE; KLEINFELD, 1991); espectrofotometria no visível (ITAYA; UI, 1965; FALHOLT; LUND; FALHOLT, 1973; LOWRY; TINSLEY, 1976; BYNUM; SENYK; BARBANO, 1984) e cromatografia (SUKHIJA; PALMQUIST, 1988; GARCIA REGUEIRO; GIBERT; DIAZ, 1994).

Todas essas metodologias possuem vantagens e desvantagens. Potenciometria é uma técnica simples, mas tem a desvantagem de ser demorada, dificultando sua utilização para um grande número de análises (TURYAN; BEREZIN; SHENHAR, 1996). As metodologias no infravermelho próximo possuem a vantagem de não haver a necessidade de preparo de um composto para ser analisado, no entanto, a desvantagem está nos espectros obtidos que são complexos e algumas vezes de difícil interpretação (ZHANG; LEE, 1997; MAN; MOH, 1998). As técnicas de espectrofotometria no visível são usualmente demoradas, pois envolvem a reação do ácido graxo com um cromóforo (FALHOLT; LUND; FALHOLT, 1973; LOWRY; TINSLEY, 1976). As metodologias envolvendo cromatografia possuem como vantagem a possibilidade de quantificar cada ácido graxo, no entanto, exigem uma etapa de preparo de um composto esterificado, o mesmo problema que ocorre com a técnica de fluorescência (SUKHIJA; PALMQUIST, 1988; STORCH; LECHENE; KLEINFELD, 1991; GARCIA REGUEIRO; GIBERT; DIAZ, 1994).

A espectroscopia no infravermelho analisa os movimentos vibracionais das moléculas/e ou grupos das mesmas. Esta técnica espectroscópica é muito utilizada no estudo da conformação, identificação e interação com outras moléculas (COLTHUP; DALY; WIBERLEY, 1964).

Nos últimos anos a espectroscopia no infravermelho vem sendo muito utilizada para fins analíticos, e está sendo muito utilizada na determinação de ácidos graxos livres em diferentes meios: extratos brutos de óleo de soja (LANSER et al., 1991), ácidos graxos em tecido adiposo de porco (RIPOCHE; GUILLARD, 2001; OLSEN et al., 2008), ácidos graxos trans em óleos comestíveis (VAN DE VOORT et al., 2008; SHERAZI et al., 2009), ácidos graxos em mucosa oral (YOSHIDA et al., 2008) e na análise de lubrificantes (VAN DE VOORT et al., 2008).

Uma das grandes vantagens da espectroscopia no infravermelho é que em algumas das metodologias propostas não há necessidade de um pré-preparo da amostra para análise, ou seja, podemos economizar grande quantidade de reagentes, reduzir o tempo e o pessoal para as análises (OLSEN et al., 2008; YOSHIDA et al., 2008; SHERAZI et al., 2009). Outra vantagem de algumas das metodologias de infravermelho é que elas não são destrutivas (OLSEN et al., 2008; YOSHIDA et al., 2008).

Assim, o objetivo deste trabalho foi desenvolver uma metodologia geral utilizando a técnica de infravermelho para a determinação de ácidos graxos livres totais.

\section{Materiais e Métodos}

\section{Reagentes}

Os seguintes reagentes foram utilizados no presente trabalho, sendo que todos são de grau analítico P.A: ferricianeto de potássio (Merck), ácido palmítico (Across), heptano (Synth), metanol (Nuclear), clorofórmio (Synth), brometo de potássio (Merck).

\section{Preparo do padrão interno}

Para cada 1,00 g de brometo de potássio foi adicionado $20 \mathrm{mg}$ de ferricianeto de potássio $\left(\mathrm{K}_{3}\left[\mathrm{Fe}(\mathrm{CN})_{6}\right]\right)$ como padrão interno, sendo que os sólidos foram misturados até a homogeneização utilizando um gral de ágata e pistilo. 


\section{Curva analítica}

Como padrão foi utilizado ácido palmítico $(2,00$ mmol $\left.\mathrm{L}^{-1}\right)$. Foram pipetados, em duplicatas, os seguintes volumes do padrão de ácido palmítico: 50, 100, 150, 200, 300, 450, 600, 750, 900 mL, então foram acrescentados $1,0 \mathrm{~mL}$ de tampão fosfato $(\mathrm{pH}$ 6,20) e $6,0 \mathrm{~mL}$ de solução extratora (clorofórmio: 45 $\mathrm{mL}$; n-heptano: $45 \mathrm{~mL}$; metanol: $10 \mathrm{~mL}$ ) e agitou-se por 2 minutos. Após 15 minutos de repouso as amostras foram centrifugadas por 20 minutos a 2.000 r.p.m., a fase aquosa foi descartada e $5 \mathrm{~mL}$ da fase orgânica foi utilizada para a análise utilizando a espectroscopia no infravemelho.

\section{Interferentes}

Para testar as possíveis substâncias interferentes do método, foram feitas análises em quadruplica, contendo $300 \mu \mathrm{L}$ de solução padrão de ácido palmítico em cada. Na solução considerada como branco, apenas o padrão foi pipetado. Nas demais, para verificar a possível interferência, foram adicionadas as seguintes medidas nas amostras: 50 e $100 \mu \mathrm{L}$ de solução contendo aminoácidos [Gly, Ala e Cys $\left(5 \mathrm{mg} \mathrm{mL}^{-1}\right)$ ]; 25 e $50 \mu \mathrm{L}$ de heparina (5000 U.I mL $\mathrm{mL}^{-1}$ ); 50 e $100 \mu \mathrm{L}$ de colesterol (2,00 $\left.\mathrm{mg} \mathrm{mL}^{-1}\right) ; 25$ e $50 \mu \mathrm{L}$ de triacilglicerol $(2,00 \mathrm{mg}$ $\left.\mathrm{mL}^{-1}\right) ; 25$ e $50 \mu \mathrm{L}$ de solução contendo cobre $(4,5 \mathrm{mg}$ $\mathrm{mL}^{-1) ;} 25$ e $50 \mu \mathrm{L}$ de solução de $\mathrm{FeCl} 3\left(4,5 \mathrm{mg} \mathrm{mL}^{-1}\right)$; 150 e $500 \mu \mathrm{L}$ de mistura de sais mistura de sais [cloreto de sódio $\left(28,56 \mathrm{~g} \mathrm{~L}^{-1}\right)$, cloreto de magnésio $(3,88 \mathrm{~g}$ $\left.\mathrm{L}^{-1}\right)$, sulfato de magnésio $\left(1,79 \mathrm{~g} \mathrm{~L}^{-1}\right)$, sulfato de cálcio $\left(1,31 \mathrm{~g} \mathrm{~L}^{-1}\right)$, sulfato de potássio $\left(0,83 \mathrm{~g} \mathrm{~L}^{-1}\right)$, brometo de potássio $\left.\left(0,1 \mathrm{~g} \mathrm{~L}^{-1}\right)\right]$; 150 e $500 \mu \mathrm{L}$ de glicose $(2,00$ $\left.\mathrm{g} \mathrm{mL}^{-1}\right)$.

\section{Espectros infravermelhos}

A solução (5,0 $\mathrm{ml}$ da fase orgânica) foi misturada com 200 mg de KBr, evaporada com a ajuda de lâmpada de 200 w e então submetida ao pastilhamento para a análise no espectro de infravermelho. Os espectros FTIR foram obtido com o equipamento Shimadzu FT-IR 8300, utilizando pastilhas de $\mathrm{KBr}$ e com uma resolução de $4 \mathrm{~cm}^{-1}$, cada espectro foi obtido após 180 aquisições. Os espectros foram obtidos na faixa de 400 até $4000 \mathrm{~cm}^{-1}$ e a absorbância foi medida considerando a diferença entre absorbância no pico menos a absorbância medida na linha base. Os espectros foram analisados utilizando o programa Origin $(5.0,2001)$.

\section{Resultados e discussão}

O ácido palmítico é um ácido graxo saturado de cadeia longa formado por 16 carbonos cuja formula química é $\mathrm{CH}_{3}\left(\mathrm{CH}_{2}\right)_{14} \mathrm{COOH}$. O mesmo foi escolhido como padrão no presente estudo visto que é o ácido graxo em maior concentração no plasma sanguíneo e também em muitos outros meios biológicos ou alimentos (CURI et al., 2002; NEY; TORRES; TRUGO, 2004).

A figura 1 mostra os espectros utilizando a espectroscopia no infravermelho do padrão do ácido palmítico e do padrão de ácido palmítico mais o padrão interno de ferricianeto de potássio. $\mathrm{O}$ ácido palmítico tem duas bandas absorção na região de 2852 e $2920 \mathrm{~cm}-1$ devido ao estiramento C-H (Figura 1-A e 1-B) (COLTHUP; DALY; WIBERLEY, 1964). O ferricianeto de potássio possui uma banda de absorção intensa em $2118 \mathrm{~cm}^{-1}$ devido ao estiramento $\mathrm{CN}$ (Figura 1-B) (COLTHUP; DALY; WIBERLEY, 1964). Ambos os espectros mostram bandas de absorção na região de $2350 \mathrm{~cm}^{-1}$ que são devido ao $\mathrm{CO} 2$ presente no ar que se altera durante a aquisição dos espectros, desta forma não são subtraídos do espectro de referência (COLTHUP; DALY; WIBERLEY, 1964). Este composto foi escolhido como padrão interno visto que na região onde o mesmo apresenta uma banda absorção intensa, o ácido palmítico não possui nenhuma banda de absorção. O sulfato de sódio também foi estudado como padrão interno, no entanto, os coeficientes de correlação da reta obtidos eram inferiores do que aqueles obtidos com ferricianeto de potássio.

A figura 2 mostra as curvas analíticas para as bandas de absorção na região de 2852 e 2920 $\mathrm{cm}^{1}$. Foram obtidas as seguintes equações da reta para as figuras 1A e 1B: $\mathrm{Y}=0,0011 \mathrm{X}+0,09[\mathrm{Y}=$ 
(Absorbância do ácido palmítico em $2851 \mathrm{~cm}^{-1} /$ Absorbância do ferricianeto de potássio em $2118 \mathrm{~cm}^{-}$ ${ }^{1}$ ), $\mathrm{X}=$ concentração do ácido palmítico em mmol L-1, coeficiente de correlação $=0,9496$ ] e $Y=0,0015$ $\mathrm{X}+0,15[\mathrm{Y}=$ (Absorbância do ácido palmítico em $2920 \mathrm{~cm}^{-1} /$ Absorbância do ferricianeto de potássio em $\left.2118 \mathrm{~cm}^{-1}\right), \mathrm{X}=$ concentração do ácido palmítico em mmol L-1, coeficiente de correlação $=0,9507]$, respectivamente. A lei de Beer-Lambert para ambas as bandas é seguida numa ampla faixa de concentração de ácido palmítico (14-257 mmol L-1) (Figura 2). Como podemos verificar, pelas equações da reta, a banda de absorção em $2920 \mathrm{~cm}^{-1}$ apresentou maior absortividade molar do que a banda de absorção em $2851 \mathrm{~cm}^{-1}$.
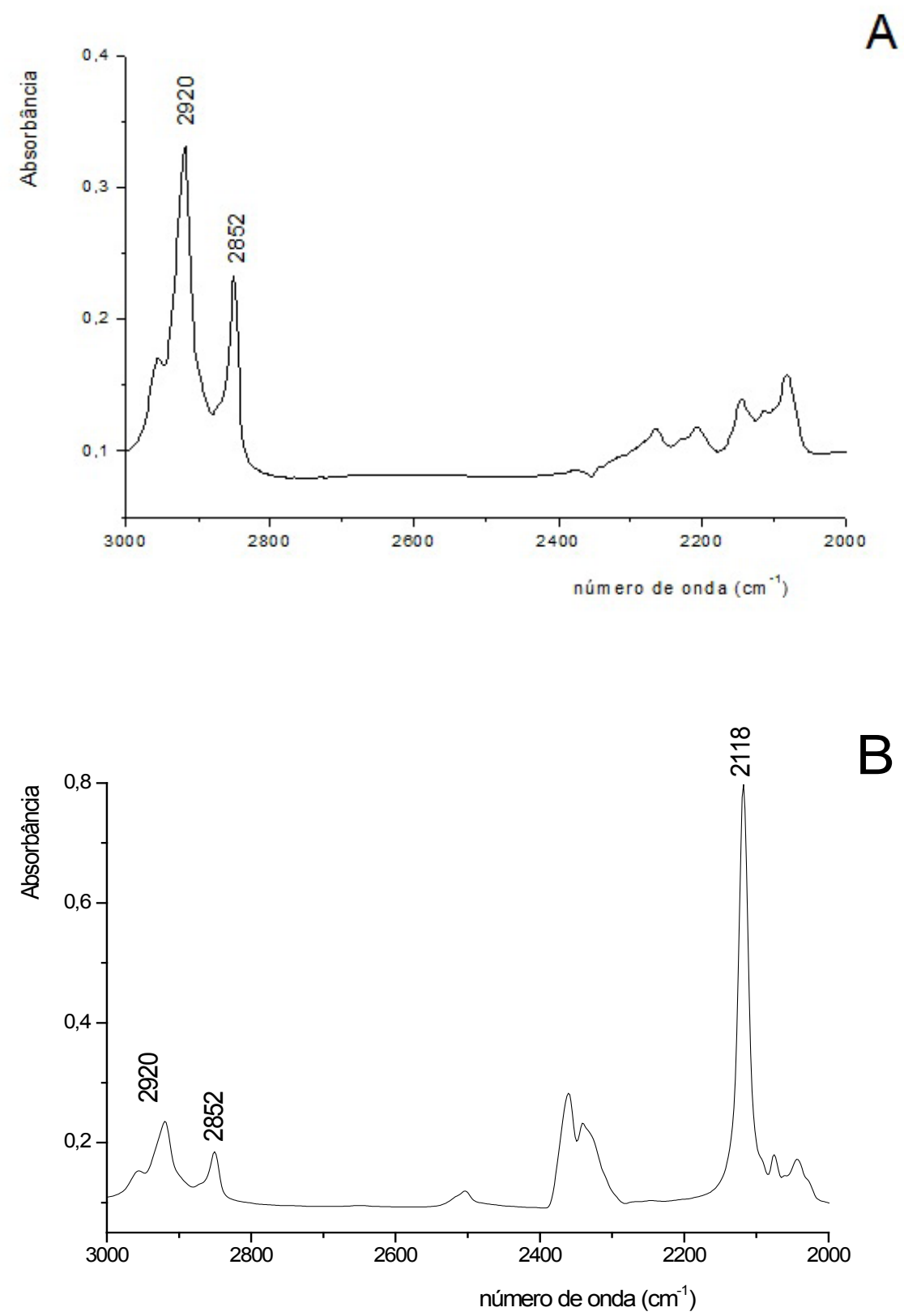

Figura 1. Espectros infravemelhos do ácido palmítico (A) e ácido palmítico mais o padrão ferricianeto de potássio (B). $\mathrm{O}$ ferricianeto de potássio $\left(\mathrm{K}_{3}\left[\mathrm{Fe}(\mathrm{CN})_{6}\right]\right)$ foi adicionado ao brometo de potássio $(\mathrm{KBr})$ na concentração de $20 \mathrm{mg}$ $\mathrm{g}^{-1}$. A concentração de ácido palmítico é de $60,0 \mu \mathrm{mol} \mathrm{L}{ }^{-1}$. 

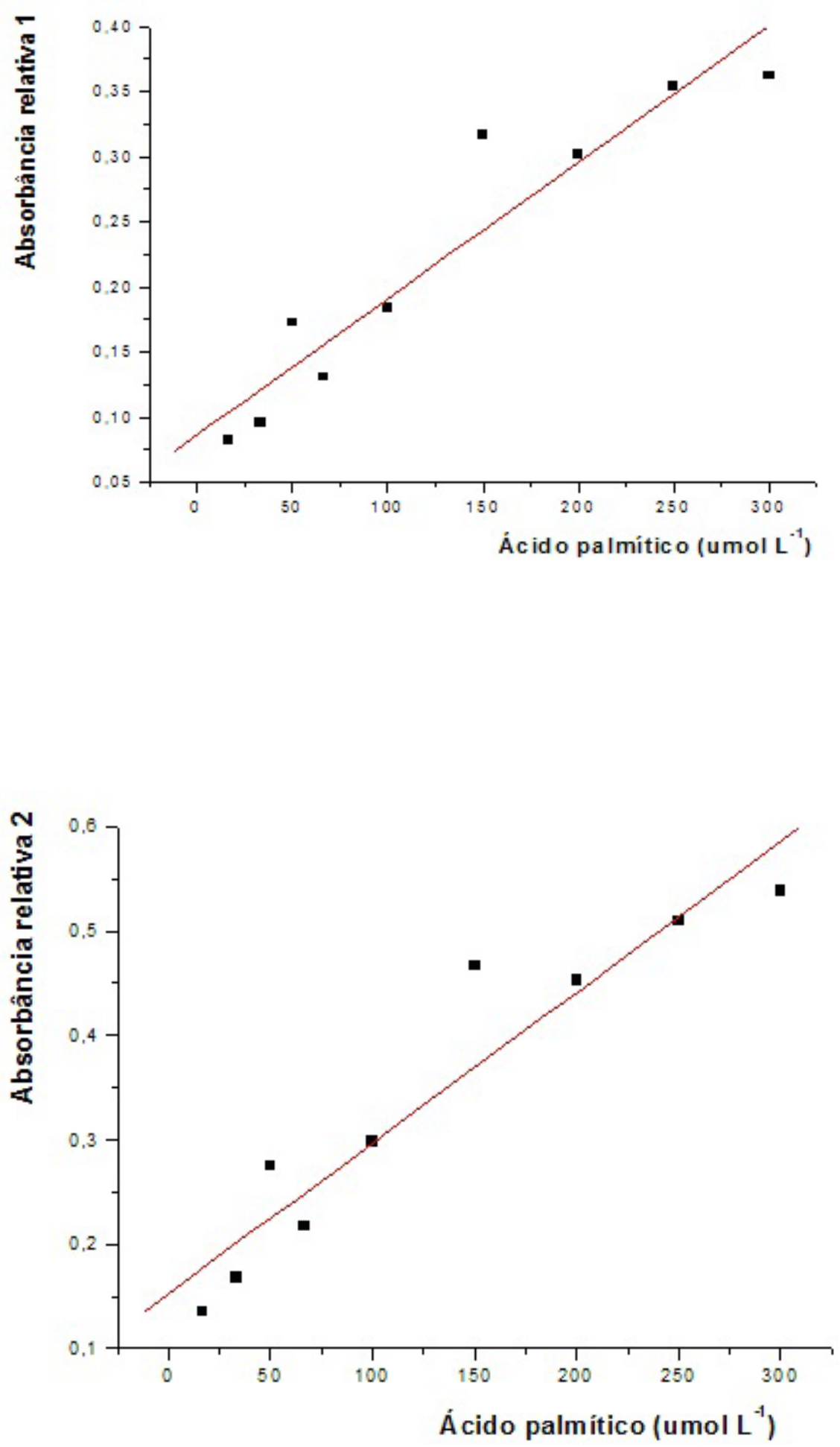

Figura 2. Curva analítica do ácido palmítico. Absorbância versus concentração de ácido palmítico, em $\mu$ mol L ${ }^{-1}$. A: Absorbância relativa 1= (Absorbância do ácido palmítico em $2851 \mathrm{~cm}^{-1} /$ Absorbância do ferricianeto de potássio em $2118 \mathrm{~cm}^{-1}$ ), B: Absorbância relativa 2= (Absorbância do ácido palmítico em $2920 \mathrm{~cm}^{-1} /$ Absorbância do ferricianeto de potássio em $2118 \mathrm{~cm}^{-1}$ ). 
A tabela 1 mostra o estudo de diversas substâncias que poderiam interferir com a metodologia proposta. Colesterol, triacilglicerol, cloreto férrico (na maior concentração) e a mistura de aminoácidos (na maior concentração para a banda de absorção em $2919 \mathrm{~cm}^{-1}$ ) foram as substâncias que apresentaram interferência para a metodologia proposta $(\mathrm{p}<0,05)$. Para o colesterol e triacilglicerol, esta interferência é devida ao fato de que estas substâncias apresentam uma banda de absorção no infravermelho próxima da banda de absorção do ácido palmítico, que foi utilizada na presente proposta (figura não mostrada); devemos salientar que as concentrações de colesterol e triacilglicerol utilizadas no presente trabalho são maiores que as encontradas no plasma sanguíneo (colesterol: $68 \mathrm{mg} / 100 \mathrm{~mL}$; triacilglicerol: $81 \mathrm{mg} / 100 \mathrm{~mL}$; WAYNFORTH, 1980), portanto, estas substâncias não serão interferentes na metodologia proposta. No caso do cloreto férrico e da mistura de aminoácidos, a interferência foi somente observada em altas concentrações.

Tendo em vista que na presente metodologia geral desenvolvida não há necessidade de um pré-preparo da amostra, como ocorre nas metodologias envolvendo a cromatografia (SUKHIJA; PALMQUIST, 1988; GARCIA REGUEIRO; GIBERT; DIAZ, 1994), e também não envolve nenhuma reação com um cromóforo como no caso das metodologias utilizando a espectroscopia no visível ou fluorescência (ITAYA; UI, 1965; FALHOLT; LUND; FALHOLT, 1973; LOWRY; TINSLEY, 1976; BYNUM; SENYK; BARBANO, 1984; STORCH; LECHENE; KLEINFELD, 1991) o presente procedimento desenvolvido é simples, barato e de fácil utilização podendo ser adaptado para inúmeras aplicações. 
Tabela 1. Efeitos dos potenciais interferentes no método.

\begin{tabular}{|c|c|c|c|}
\hline $\begin{array}{l}\text { AGL }\left(2 \text { mmol L }^{-1}\right)+ \\
\text { interferente }\end{array}$ & Adição & $\begin{array}{c}\text { *Absorbância em } \\
2852 \mathrm{~cm}^{-1} \mathrm{em} \\
\text { porcentagem }\end{array}$ & $\begin{array}{c}* \text { Absorbância em } \\
2919 \mathrm{~cm}^{-1} \mathrm{em} \\
\text { porcentagem }\end{array}$ \\
\hline $\begin{array}{l}\text { Padrão AGL } \\
\left(2 \mathrm{mmol} \mathrm{L}^{-1}\right)\end{array}$ & Branco & $100,0 \pm 5,6^{\mathrm{a}}$ & $100,0 \pm 5,9$ \\
\hline \multirow{2}{*}{ Aminoácidos } & $50 \mu \mathrm{L}$ & $120,0 \pm 18,3$ & $119,4 \pm 17,3$ \\
\hline & $100 \mu \mathrm{L}$ & $127,8 \pm 16,3$ & $142,6 \pm 15,7^{b}$ \\
\hline \multirow{2}{*}{ Heparina } & $25 \mu \mathrm{L}$ & $77,6 \pm 22,1$ & $76,8 \pm 20,4$ \\
\hline & $50 \mu \mathrm{L}$ & $105,8 \pm 7,0$ & $97,1 \pm 0,8$ \\
\hline \multirow{2}{*}{ Colesterol } & $50 \mu \mathrm{L}$ & $538,8 \pm 4,2^{b}$ & $310,7 \pm 35,7^{b}$ \\
\hline & $100 \mu \mathrm{L}$ & $955,0 \pm 8,4^{b}$ & $735,1 \pm 12,7^{b}$ \\
\hline \multirow{2}{*}{ Triacilglicerol } & $25 \mu \mathrm{L}$ & $53,1 \pm 12,1^{b}$ & $53,8 \pm 11,5^{b}$ \\
\hline & $50 \mu \mathrm{L}$ & $67,8 \pm 10,8^{b}$ & $69,4 \pm 9,7^{b}$ \\
\hline \multirow{2}{*}{ Cloreto de cobre } & $25 \mu \mathrm{L}$ & $102,1 \pm 19,1$ & $99,5 \pm 21,9$ \\
\hline & $50 \mu \mathrm{L}$ & $96,7 \pm 12,2$ & $98,6 \pm 12,4$ \\
\hline \multirow{2}{*}{ Cloreto férrico } & $25 \mu \mathrm{L}$ & $126,5 \pm 8,7$ & $122,9 \pm 9,4$ \\
\hline & $50 \mu \mathrm{L}$ & $127,3 \pm 6,2^{b}$ & $128,2 \pm 5,9^{b}$ \\
\hline \multirow{2}{*}{ Mistura de Sais } & $150 \mu \mathrm{L}$ & $85,3 \pm 11,7$ & $85,1 \pm 11,7$ \\
\hline & $500 \mu \mathrm{L}$ & $151,3 \pm 39,4$ & $143,3 \pm 37,4$ \\
\hline \multirow{2}{*}{ Glicose } & $150 \mu \mathrm{L}$ & $88,5 \pm 9,8$ & $89,7 \pm 9,8$ \\
\hline & $500 \mu \mathrm{L}$ & $87,4 \pm 18,6$ & $89,5 \pm 19,7$ \\
\hline
\end{tabular}

Os resultados apresentados são expressos como média \pm erro padrão da média de quatro amostras. *Todas as absorbâncias foram relativas ao padrão interno de ferricianeto de potássio. AGL = ácido graxo livre (ácido palmítico); aminoácidos: Gly, Ala e Cys (5 mg mL $\left.\mathrm{m}^{-1}\right)$; heparina (5000 U.I mL $\left.\mathrm{m}^{-1}\right)$; colesterol (2,00 mg mL $\left.\mathrm{mL}^{-1}\right)$; triacilglicerol (2,00 $\left.\mathrm{mg} \mathrm{mL}^{-1}\right)$; cloreto de cobre $\left(4,5 \mathrm{mg} \mathrm{mL}^{-1}\right)$; cloreto férrico $\left(4,5 \mathrm{mg} \mathrm{mL}^{-1}\right)$; mistura de sais: cloreto de sódio $\left(28,56 \mathrm{~g} \mathrm{~L}^{-1}\right)$, cloreto de magnésio $\left(3,88 \mathrm{~g} \mathrm{~L}^{-1}\right)$, sulfato de magnésio $\left(1,79 \mathrm{~g} \mathrm{~L}^{-1}\right)$, sulfato de cálcio $\left(1,31 \mathrm{~g} \mathrm{~L}^{-1}\right)$, sulfato de potássio $\left(0,83 \mathrm{~g} \mathrm{~L}^{-1}\right)$, brometo de potássio $\left(0,1 \mathrm{~g} \mathrm{~L}^{-1}\right)$ e glicose $\left(2,00 \mathrm{~g} \mathrm{~mL}^{-1}\right)$. Teste t-Student valores estatisticamente diferentes $\mathrm{a} / \mathrm{b}(\mathrm{p}<0,05)$.

\section{Conclusões}

O ácido palmítico possui duas bandas de absorção (2852 e $2920 \mathrm{~cm}^{-1}$ ), devido ao estiramento $\mathrm{CH}$, que podem ser utilizadas para fins analíticos. $\mathrm{O}$ ferricianeto de potássio mostrou-se adequado como padrão interno. A lei de Beer-Lambert, para ambas as bandas de absorção, é seguida numa ampla faixa de concentração de ácido palmítico. A metodologia proposta está sujeita à interferência de altas concentrações de colesterol ou triacilgliceróis. $\mathrm{O}$ método proposto é barato, rápido não exigindo o pré preparo ou derivatização da amostra. 


\section{Agradecimentos}

Os autores (2) agradecem à Bolsa e Iniciação Científica do CNPq (PIBIC) e da Fundação Araucária, respectivamente.

\section{Referências}

BYNUM, D. G.; SENYK, G. F.; BARBANO, D. M. Determination of free fatty acid content of cheddar cheese by a copper soap method. Journal of Dairy Science, Lancaster, v. 67, p. 1521-1524, 1984.

COLTHUP, N. B.; DALY, L. H.; WIBERLEY, S. E. Introduction to infrared and raman spectroscopy. New York: Academic Press, 1964.

CURI, R.; POMPÉIA, C.; MIYASAKA, C. K.; PROCOPIO J. Entendendo a gordura: os ácidos graxos. Barueri: Manole, 2002.

FALHOLT, K.; LUND, B.; FALHOLT, W. An easy colorimetric method for routine determination of free fat acids in plasma. Clinica Chimica Acta, Amsterdam, v. 46, p. 105-111, 1973.

GARCIA REGUEIRO, J. A.; GIBERT, J.; DIAZ, I. Determination of neutral lipids from subcutaneous fat of cured ham by capillary gas chromatography and liquid chromatography. Journal of Chromatography, Amsterdam, v. 667, p. 225-233, 1994.

ITAYA, K.; UI, M. Colorimetric determination of free fatty acids in biological fluids. Journal of Lipid Research, Memphis, v.6, p. 16-20, 1965.

LANSER, A. C.; LIST, G. R.; HOLLOWAY, R. K.; MOUNTS, T. L. FT-IR estimation of free fatty acid content in crude extracted from damaged soybeans. Journal of the American Oil Chemists Society, Champaign, v. 6, p. 448449, 1991.

LOWRY, R. R.; TINSLEY, I. J. Rapid colorimetric determination of free fatty acids. Journal of the American Oil Chemists Society, Champaign, v. 53, p. 470-472, 1976.

MAN, B. C.; MOH, M. H. Determination of free fatty acids in palm oil by near infrared reflectance spectroscopy. Journal of the American Oil Chemists Society, Champaign, v. 75, p. 557-562, 1998.

NEY, J. G.; TORRES, A. G.; TRUGO, N. M. F. Análise de ácidos graxos não esterificados de plasma humano por cromatografia gasosa capilar com injeção sem divisão de fluxo. Química Nova, São Paulo, v. 27, p. 561-566, 2004.

OLSEN, E. J.; RUKKE, E. O.; EGELANDSDAL, B.; ISAKSSON, T. Determination of omega-6 and omega-3 fatty acids in pork adipose tissue with nondestructive Raman and Fourier transform infrared spectroscopy. Applied Spectroscopy, Plainfield, v. 62, p. 968-974, 2008.

RIPOCHE, A.; GUILLARD, A. S. Determination of fatty acid composition of pork fat by Fourier transform infrared spectroscopy. Meat. Science, Oxford, v. 58, p. 299-304, 2001.

SHERAZI, S. T. H.; KANDHRO, A.; MAHESAR, S. A.; BHANGER, M. I.; TALPUR, M. Y.; ARAIN, S. Application of transmission FT-IR spectroscopy for the trans fat determination in the industrially processed edible oils. Food Chemistry, Oxford, v. 114, p. 323-327, 2009.

STORCH, J.; LECHENE, C.; KLEINFELD, A. M. Direct determination of free fatty acid transport across the adipocyte plasma membrane using quantitative fluorescence microscopy. Journal of Biological Chemistry, v. 266, p. 13473-13476, 1991.

SUKHIJA, P. S.; PALMQUIST, D. L. Rapid method for determination of total fatty acid content and composition of feedtuffs and feces. Journal of Agricultural Food and Chemistry, v. 36, p. 1202-1206, 1988.

TURYAN, Y.I.; BEREZIN, O.Y.; SHENHAR, A. pH metric determination of acid values in vegetable oils without titration. Journal of the American Oil Chemists Society, v. 73, p. 295-301, 1996.

VAN DE VOORT, F. R.; GHETLER, A.; GARCIAGONZÁLEZ, D. L.; LI, Y. D. Perspectives on quantitative mid-FT-IR spectroscopy in relation to edible oil and lubricant analysis: evolution and integration of analysis methodologies. Food and Analytical Methods, v. 1, p. 153163, 2008.

WAYNFORTH, H.B. Experimental and surgical technique in the rat. New York: Academic Press, 1980.

YOSHIDA, S.; OKAZAKI, Y.; YAMASHIDA, T.; UEDA, H.; GHADIMI, R.; HOSONO, A.; TANAKA, T.; KURIKI, K.; SUZUKI, S.; TOKUDOME, S. Analysis of human oral mucosa ex vivo for fatty acid compositions using Fourier transform infrared spectroscopy. Lipids, v. 43, p. 361-372, 2008.

ZHANG, H. Z.; LEE, T. C. Rapid near infrared spectroscopic method for the determination of free fatty acid in fish and its application in fish quality assessment. Journal of Agricultural and Food Chemistry, v. 45, p. 3515-3521, 1997.

Recebido em 25 Setembro 2010 - Received on September 25, 2010.

Aceito em 31 Março, 2011 - Accepted on March 31, 2011. 